\title{
DESIGN AND FABRICATION OF AN ACHROMATIC BEND FOR MEDIUM ENERGY ELECTRON COOLING AT FERMILAB
}

\author{
M.W. McGee, P.G. Hurh and K.J. Bertsche* \\ Fermi National Accelerator Laboratory, Batavia, Illinois $60510^{\dagger}$
}

\begin{abstract}
The use of an achromatic bend for a medium energy electron cooling beamline in the new Recycler Ring at Fermilab is required to ensure a stable beam position. This first order $180^{\circ}$ achromat consists of two $90^{\circ}$ dipoles with a $61 \mathrm{~cm}$ bend radius and a central dispersionsuppressing quadrupole flanked symmetrically by a weak solenoid and an air-core $\cos (2 \theta)$ quadrupole. The mechanical design and technique of fabrication for each bend component is discussed. Each of the air cooled mechanical designs are simple yet precise in terms of construction technique. The results of magnetic field measurements are also presented.
\end{abstract}

\section{INTRODUCTION}

Medium energy electron cooling for the Recycler Ring requires a highly parallel electron beam with less than 20 $\mu \mathrm{rad}$ divergence. The beam transport system must be carefully designed and constructed to preserve these parameters. Achromatic bends have been adopted, and these are designed so that components and beam profiles are symmetric about the midpoint.

A simple first-order achromatic bend is achieved by splitting the bend equally in two bend magnets and placing a quadrupole lens between them[1]. The quadrupole cancels dispersion by imaging the center of the first bend magnet onto the center of the second. This places the two bend magnets $180^{\circ}$ apart in horizontal betatron phase and causes particles of different energies to emerge at the same point and with the same angle, to first order.

Achromatic bends not only stabilize the trajectory against energy spreads or energy fluctuations in the beam, they also stabilize the trajectory against power supply fluctuations (assuming magnets separated by $180^{\circ}$ are operated on common supplies). For a relativistic beam, achromatic transport is also isochronous. This is essential for electron cooling if a recirculating electrostatic accelerator is used. Otherwise, energy fluctuations would affect the timing and modulate the instantaneous beam current, tending to destabilize the system.

For beam symmetry about the midpoint of the system, there should be a vertical waist at the center of the central quadrupole. Most of the vertical focusing to accomplish this comes from the edges of the dipole magnets. Additional small adjustments of vertical and horizontal focusing are provided at each end of the achromat. Because of the small lens strength required,

*Currently with Siemens Medical Systems, Concord, California 94520.

${ }^{\dagger}$ Work supported by the US Department of Energy under contract number DE-AC02-76CHO3000. weak solenoid lenses and $\cos (2 \theta)$ quadrupoles are used. Figure 1 displays the layout for the achromat.

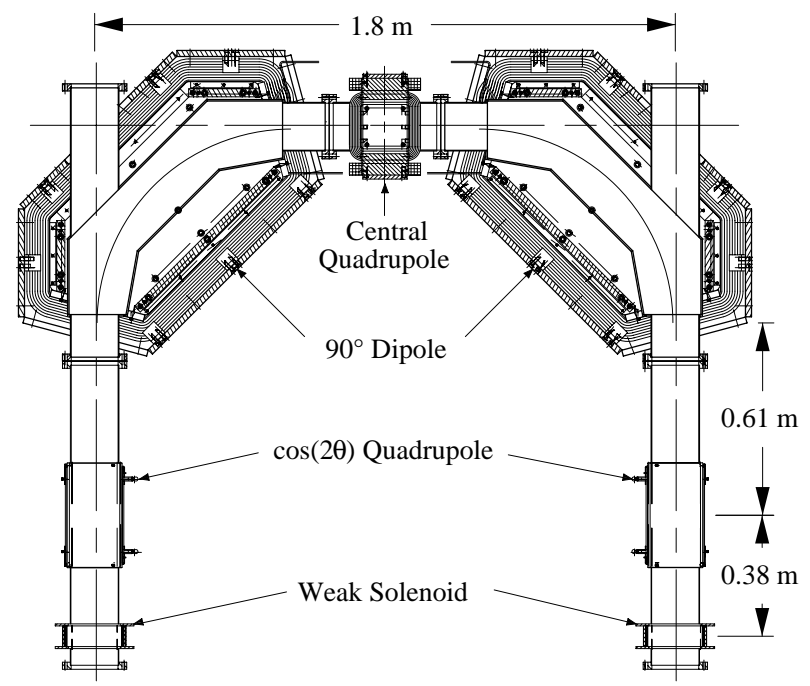

Figure 1. Top view of achromat.

\section{MECHANICAL DESIGN}

\section{$2.190^{\circ}$ Dipoles}

The $90^{\circ}$ dipoles are designed to satisfy several magnetic and geometric criteria. For a bend radius of 61 $\mathrm{cm}$ and a $5 \mathrm{MeV}$ electron beam, a 300 Gauss field is required. In addition, in order to counter the strong horizontal focusing associated with a $90^{\circ}$ dipole, the use of edge angles on the pole ends is desired. For a total beam separation of $1.8 \mathrm{~m}$ between the entry and exit beam lines, a $15^{\circ}$ edge angle provides nearly enough focusing to focus the beam both vertically and horizontally at the center of the dispersion-suppressing central quadrupole. The body field of each dipole is desired to be as uniform as practically possible (less than $\pm 0.5 \%$ variation) and the beam trajectory field integral of both dipoles are desired to match each other within $\pm 0.1 \%$ to ensure symmetry of the bend when dipoles are run in series on the same power supply.

To accommodate electron cooling in the Recycler Ring, the vacuum chambers in the dipoles must allow for a $15.2 \mathrm{~cm}$ diameter aperture. This sets the dipoles' pole tip gap at $16.5 \mathrm{~cm}$ (allowing for vacuum chamber wall thickness). In addition the vacuum chambers must provide an entrance/exit path for the cooled ion beam. This requires a large opening in the flux return steel of the dipoles.

Figure 2 shows an end view of a dipole magnet. The $90^{\circ}$ dipoles are designed with a simple, bolted together 
construction method using solid steel (1010) plates rather than laminations. The pole pieces are $5.1 \mathrm{~cm}$ thick and flux return pieces are $1.9 \mathrm{~cm}$ thick. All pieces are ground flat to $50 \mu \mathrm{m}$ where they mate together and on the pole faces. The pole plates are spaced apart with precisely machined brass blocks featuring dowel pins to align the pole pieces to each other within $100 \mu \mathrm{m}$. The ends of the magnet feature field clamps to control the field fall-off and removable steel pieces which may be re-machined to eliminate some of the second order aberration terms. Small field shim strips are also added to the edges of the pole pieces to ensure flat field across the aperture.

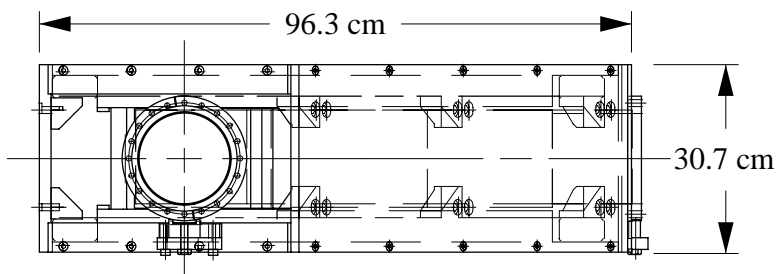

Figure 2. End view of $90^{\circ}$ dipole magnet.

Coils are constructed of 119 turns of \#6 magnet wire layered with epoxy resin tape. They were cured at $149^{\circ} \mathrm{C}$ for several hours and then wrapped with glass fiber tape and painted with room temperature curing epoxy. The coils fit tightly around the pole pieces and are secured in place with several brass brackets.

The completed dipole pole faces were mechanically measured using a coordinate axes measurement machine and found to be parallel to each other within $180 \mu \mathrm{m}$ in the worst case. This is equivalent to $0.1 \%$ of the magnet aperture.

The field variation across the aperture of the dipoles was measured using a conventional hall probe. Figure 3 shows the variation at the centers of the magnets to be less than $0.14 \% \pm 0.12 \%$ of the full field (303 Gauss at $17.36 \mathrm{~A})$. Figure 4 shows a typical field fall-off for a magnet end along the beam trajectory. Using the measured field integrals along the beam trajectory, the effective lengths of the magnets are found to differ by $0.38 \%$. This difference can be reduced in practice by using a shunt to regulate the current in one of the dipoles.

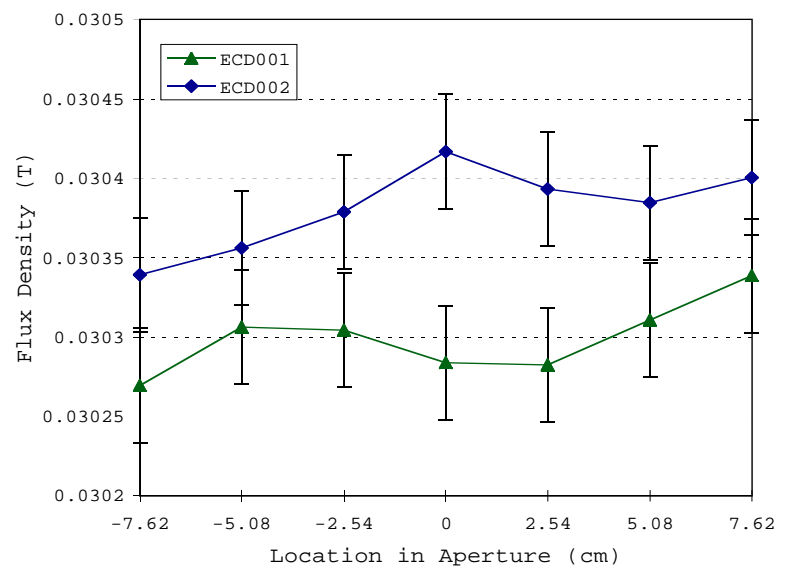

Figure 3. Field versus transverse position at dipole center.

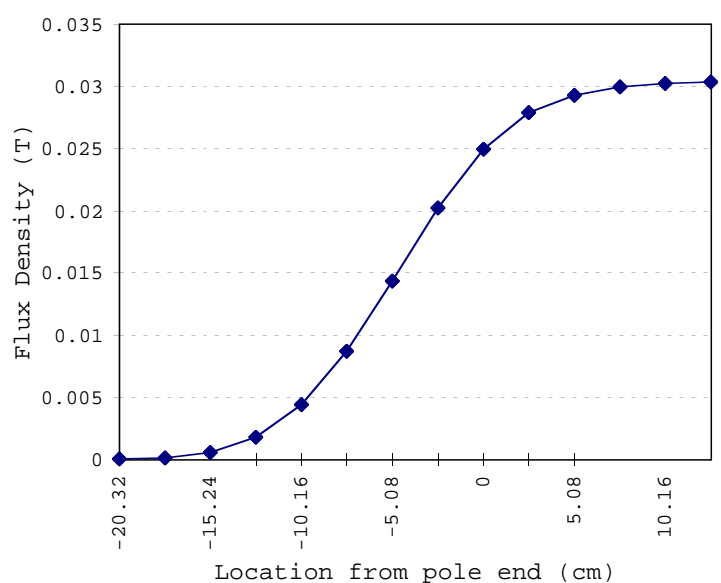

Figure 4. Typical field fall-off for $90^{\circ}$ dipole magnet.

\subsection{Central Quadrupole}

Due to space constraints, the central quadrupole is very short, with a ratio of bore diameter to iron of 1.22. A pole tip field strength of 400 Gauss is required, which corresponds to a gradient of $0.469 \mathrm{~T} / \mathrm{m}$. The steel structure of the quadrupole consists of 4 yoke plates and pole pieces made of (1018) and (1008) steel, respectively. The pole tips have a circular cross-section, with a diameter 1.15 times the aperture diameter to eliminate the 12-pole component of the body field[2]. Figure 5 shows a combined cross-section and end view of the central quadrupole.

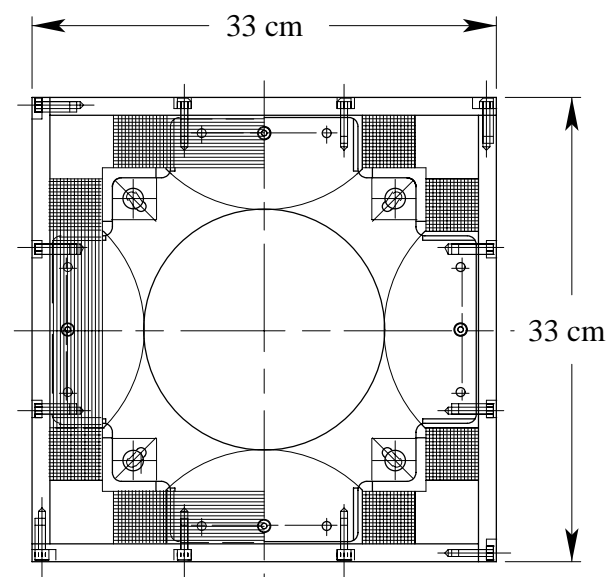

Figure. 5 End view of central quadrupole.

To eliminate the 12-pole component of the end fields, each pole was corrected with a $45^{\circ}$ chamfer as described by Hassenzahl[3]. The integrated multipole fields were measured with a Morgan coil before trimming, after trimming one end, and finally after the other end was trimmed. The second measurement confirmed the correctness of the chamfer depth. The chamfer chord length divided by bore diameter was 0.097 .

Stainless steel alignment plates located on each end ensured the repeatability of quadrupole assembly. The placement of each pole piece was indexed from one 
reference dowel pin location with respect to the bore center. The dual alignment plate feature of the mechanical design allowed for an iterative process of pole trimming described above. An alignment mandrel was placed in the center to ensure relative pole positioning during initial assembly and assist in pole end trimming.

Each pole piece was ground flat on five surfaces and each pole radius was cut using an electrodischarge machining (EDM) process with a $250 \mu \mathrm{m}$ diameter wire. The yoke and alignment plates were also ground to flatness within $25 \mu \mathrm{m}$.

Each coil consists of 225 turns of \#12 solid square conductor. Between each layer an epoxy resin filled tape provides added insulation. The coils were cured over a duration of three hours in a $177^{\circ} \mathrm{C}$ oven. The design gradient was obtained with $4.5 \mathrm{~A}$.

The mechanical design could be improved by increasing the thickness of each alignment plate to maintain dimensional stability. The results of the magnetic measurements of the integrated fields at $25 \mathrm{~mm}$ provided in Table 1 show the virtual elimination of the 12-pole component $\mathrm{B}_{6}$. The sextuple $\mathrm{A}_{3}$ is higher than anticipated but adequate for present needs; the source is presumably effects of an asymmetric support stand which uses some ferromagnetic steel.

Table 1. Results of quadrupole magnetic measurement.

\begin{tabular}{|c|c|}
\hline$N=\int B_{2} d l[T]$ & $6.545 \mathrm{e}-2$ \\
\hline $\int A_{3} d l / N[1 / m]$ & $-3.920 \mathrm{e}-4$ \\
\hline $\int B_{6} d l / N\left[1 / m^{4}\right]$ & $-5.282 \mathrm{e}-5$ \\
\hline
\end{tabular}

\section{$2.3 \cos (2 \theta)$ Quadrupole}

Weak quadrupoles in the non-dispersive region outside the bend are required to provide some focus adjustment both vertically and horizontally. The effective pole tip field for these quads is so weak (20 Gauss) that remnant fields in the iron of traditionally designed quadrupoles could be problematic. Thus the outer quadrupoles are constructed without iron pole tips using wire conductors alone lying on a circular cross-section with a $\cos (2 \theta)$ density distribution.

Figure 6 shows an end view of the weak quadrupole design. Wire conductors (\#12 magnet wire) are pressed into grooves in the outer surface of a precision machined nylon tube. The wires are arranged into 4 coils of 6 turns each on a diameter of $16.5 \mathrm{~cm}$. The act of pressing the wire into the grooves actually straightens the relatively thin conductor to within $\pm 30 \mu \mathrm{m}$. The assembled coils are inserted into a steel shielding tube $(30.5 \mathrm{~cm}$ long by $0.5 \mathrm{~cm}$ wall thickness), and the coil ends are wrapped back over the edges of the steel to shield the aperture from the coil end effects. Alignment fiducials are attached to the outside of the steel shielding tube for use in aligning the quads to the beam line. For a magnet of this design, an excitation current of 12 A should develop a gradient of $0.024 \mathrm{~T} / \mathrm{m}$.

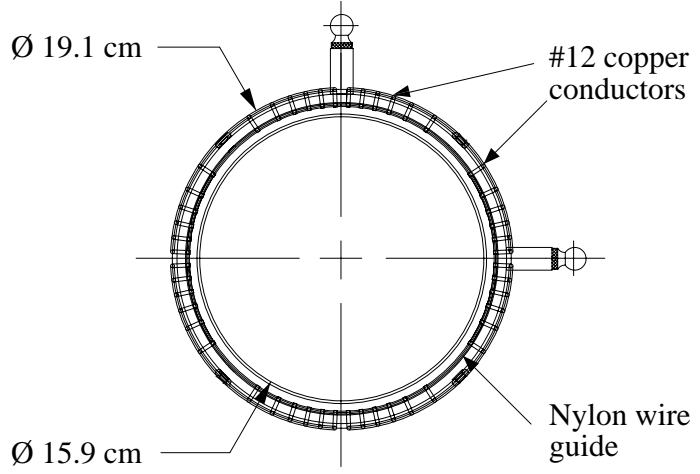

Figure 6. End view of $\cos (2 \theta)$ weak quadrupole.

Two weak quadrupoles of this design have been constructed and are currently undergoing magnetic measurements. It should be noted that because of the very weak nature of the desired gradient, stray fields are very problematic.

\subsection{Weak Solenoid}

The weak solenoid's bore diameter is $17.2 \mathrm{~cm}$ and 7.6 $\mathrm{cm}$ in length, enclosed by $6 \mathrm{~mm}$ thick permeable iron. The solenoid coil is made of \#14 square conductor with 300 turns. This design will provide a focal length of 100 $\mathrm{m}$ when excited with $5 \mathrm{~A}$.

\section{CONCLUSIONS}

The magnetic measurement results to date indicate the design success of those components tested. Also, the sensitivity of the magnetic tests to the surrounding environment demonstrates that great care in shielding the beam line and all components will be required. This bend is designed for medium energy electron cooling at Fermilab and will be tested using a proton analog beam during September of 1997.

\section{ACKNOWLEDGMENTS}

Thanks to Jim MacLachlan for his help. Technical support for the device was given by Mike Frett, Fred Saffrahn, Jim Wilson and Chih-Wen Yang.

\section{REFERENCES}

[1] John J. Livingood, "The Optics of Dipole Magnets," Academic Press, New York, 1969.

[2] A. P. Banford, "The Transport of Charged Particle Beams," London:Spon, 1966

[3] W. V. Hassenzahl, "Proceedings of 4th International Conference of Magnet Technology," BNL, 1972. 